BNL-77556-2007-CP

\title{
Superconducting non-scaling FFAG gantry for carbon/proton cancer therapy
}

\author{
D. Trbojevic, R. Gupta, B. Parker, BNL, Upton, NY 11973 USA \\ E. Keil, CERN, Geneva, Switzerland \\ A.M. Sessler, LBNL, Berkeley, CA, USA
}

Presented at the $22^{\text {nd }}$ Particle Accelerator Conference (PAC)

Albuquerque, New Mexico

June $25-29,2007$

\author{
Collider-Accelerator Department \\ Brookhaven National Laboratory \\ P.O. Box 5000 \\ Upton, NY 11973-5000 \\ www.bnl.gov
}

Notice: This manuscript has been authored by employees of Brookhaven Science Associates, LLC under Contract No. DE-AC02-98CH10886 with the U.S. Department of Energy. The publisher by accepting the manuscript for publication acknowledges that the United States Government retains a non-exclusive, paid-up, irrevocable, world-wide license to publish or reproduce the published form of this manuscript, or allow others to do so, for United States Government purposes. 


\section{DISCLAIMER}

This report was prepared as an account of work sponsored by an agency of the United States Government. Neither the United States Government nor any agency thereof, nor any of their employees, nor any of their contractors, subcontractors, or their employees, makes any warranty, express or implied, or assumes any legal liability or responsibility for the accuracy, completeness, or any third party's use or the results of such use of any information, apparatus, product, or process disclosed, or represents that its use would not infringe privately owned rights. Reference herein to any specific commercial product, process, or service by trade name, trademark, manufacturer, or otherwise, does not necessarily constitute or imply its endorsement, recommendation, or favoring by the United States Government or any agency thereof or its contractors or subcontractors. The views and opinions of authors expressed herein do not necessarily state or reflect those of the United States Government or any agency thereof. 


\title{
SUPERCONDUCTING NON-SCALING FFAG GANTRY FOR CARBON/PROTON CANCER THERAPY*
}

\author{
D. Trbojevic, R. Gupta, and B. Parker, BNL, Upton, New York 11973, USA \\ E. Keil, CERN, Geneva, Switzerland, A.M. Sessler, LBNL, Berkeley CA, USA
}

\begin{abstract}
We report on improvements in the non-scaling Fixed Field Alternating Gradient (FFAG) gantry design. As we previously reported [1], a major challenge of the carbon/proton cancer therapy facilities is isocentric gantry design. The weight of the isocentric gantry transport elements in the latest Heidelberg carbon/proton facility is 135 tons**. In this report we detail improvements to the previous non-scaling gantry design. We estimate that this non-scaling FFAG gantry would be almost hundred times lighter than traditional heavy ion gantries. Very strong focusing with small dispersion permits passage of different energies of carbon beams through the gantry's fixed magnetic field.
\end{abstract}

\section{INTRODUCTION}

The number of cancer hadron therapy facilities is rapidly growing all around the world. Many more are being commissioned or in process of being built (Germany, Italy, Japan, USA, etc.). This progress is mostly due to multiple advantages of the hadron cancer therapy with respect to any other radiation methods [2]. A major challenge in present and future hadron therapy facilities is the beam delivery system. An isocentric gantry system is becoming a necessity for each facility. The request comes mostly from hard to reach tumors especially around the spine (chordomas, low grade chondro-sarcomas, unresectable sacral chordomas). The hadron therapy treatment requires different incident angles to avoid the spine damage by radiation. The gantry role is to deliver a precise ion dose to the patients with very good reliability and stability. The larger cancerous tumors require transverse position scanning at different beam energies and an angle variation around the patient provided by gantry rotation. The non-scaling FFAG (NS-FFAG) concept should dramatically reduce overall weight [4] of the carbon gantry made of warm magnets. The fixed field magnets have transversely linear variation of the magnetic field and they could be superconducting or high temperature superconductors.

\section{Study of a gantry with smaller energy range}

This report is an update -on the previous carbon/proton design [1]. Design differences in this study come mostly due to possibility of fast magnetic field variation with a different superconductor. The superconductor wire is comprised of a multiplicity of filaments of niobium/titanium superconductor alloy disposed within a matrix of copper. The "eddy" current problem of the superconducting cables is significantly reduced and a current variation of $30 \mathrm{~A} / \mathrm{s}$ has been achieved.

In addition to the advantage of dramatically smaller weight of the NS-FFAG magnets we have previously emphasized an ability of operating the gantry at the fixed magnetic field for the whole range of carbon/proton energies required for the patient treatment. Our latest report [1] has the NS-FFAG gantry with $250-400 \mathrm{MeV}$ range, under the fixed magnet current. This corresponds to the momentum range of $\delta p / p=-25 \%$ up to $30 \%$. In the present report the momentum range is reduced to $\delta p / p=+-15 \%$. This corresponds to the kinetic energy between 235-400 MeV/u with the value of $B_{\max } \rho=5.5192819 \mathrm{Tm}$. A switch from $B_{\max } \rho$ to the new value of $B_{\text {new }} \rho=4.079469 \mathrm{Tm}$ can be done as fast as the present warm temperature magnets can do $(30 \mathrm{~A} / \mathrm{s})$. The patient treatment with a range in kinetic energy of 135$400 \mathrm{MeV}$ is now provided with two current settings.

\section{THE NON-SCALING FFAG}

The non-scaling FFAG is made of fixed field combined function magnets with a linear transverse variation of magnetic field [5].

\section{The basic cell of the Gantry}

An example a gantry basic cell with betatron functions is shown in Fig. 1.

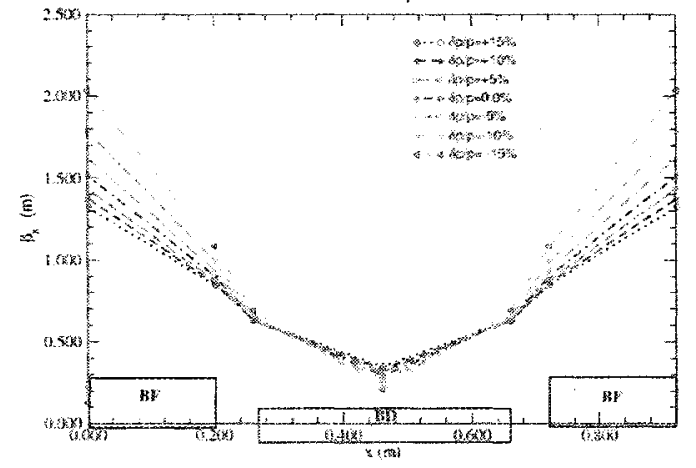

Fig. 1: Betatron functions in one gantry cell

The central magnet is a defocusing combined function magnet with minima of dispersion and horizontal $\beta_{x}$ function at the middle. Dispersion function throughout FFAG lattice retains very small values. The large momentum acceptance and small orbit offsets are a

\footnotetext{
Work performed under the United States Department of Energy

Contract No. DE-AC02-98CH1-886
} 
consequence of the small dispersion. The gantry, made of the non-scaling FFAG cells, accepts and propagates different energies ions with very small variation of the orbit. The combined function magnets of the basic cell with bending angles are presented in Fig. 2 . The middle of the dipole is selected as a place for the input and output of the gantry.

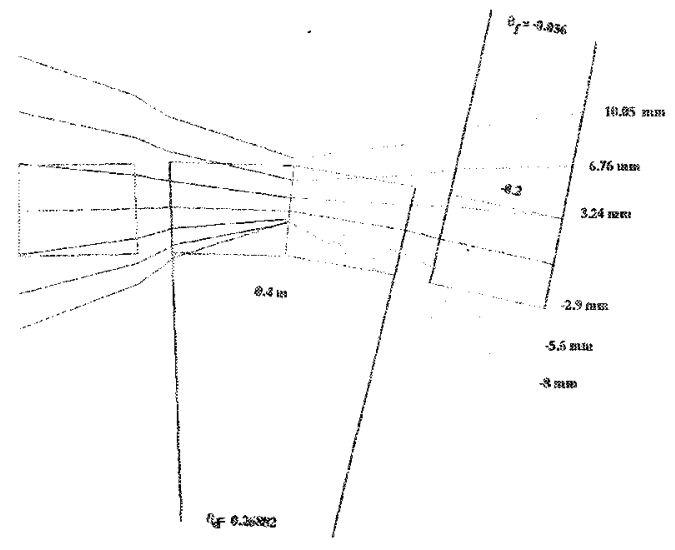

Fig. 2: The basic cell of the gantry made of non-scaling FFAG combined function magnets.

The offsets at the end of the cell are obtained from the Polymorphic Tracking Code (PTC) [6] in a kinetic energy range between in momentum $\delta p / p< \pm 15 \%$ or in kinetic energy range $131.6-400 \mathrm{MeV} / \mathrm{n}$.

\section{Gantry design}

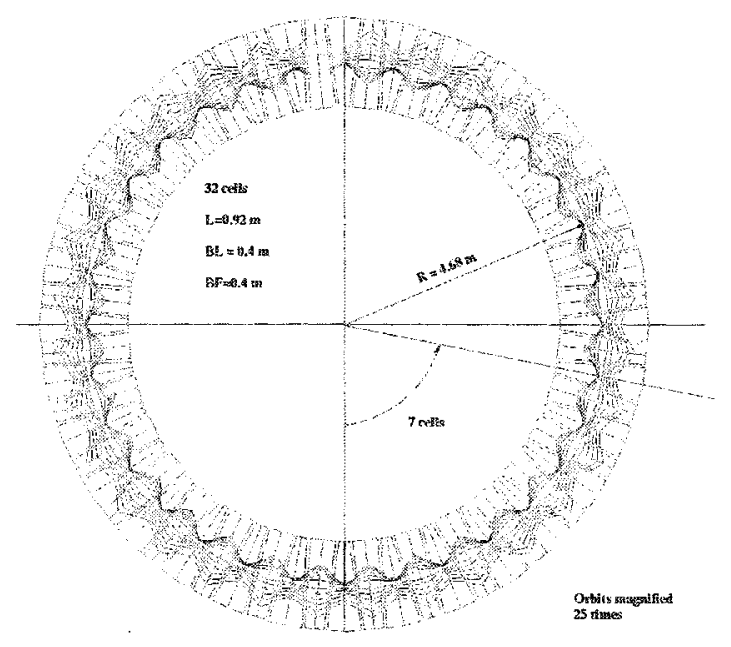

Fig. 3: Non-scaling FFAG ring to be used for the gantry design. Orbit offsets are magnified 25 times.

A design of the gantry requires a ring with zero dispersion and slopes at the middle of the major combined function dipoles as presented in Fig. 3. Stable orbits for carbon ions are found within $\delta p / p= \pm 15 \%$. The radial scanning and orbit correction is provided at the end of the gantry. A construction of the gantry follows the ring solution and 7 cells of the ring are used in the first example for the beginning of the gantry, as presented in Fig. 4.

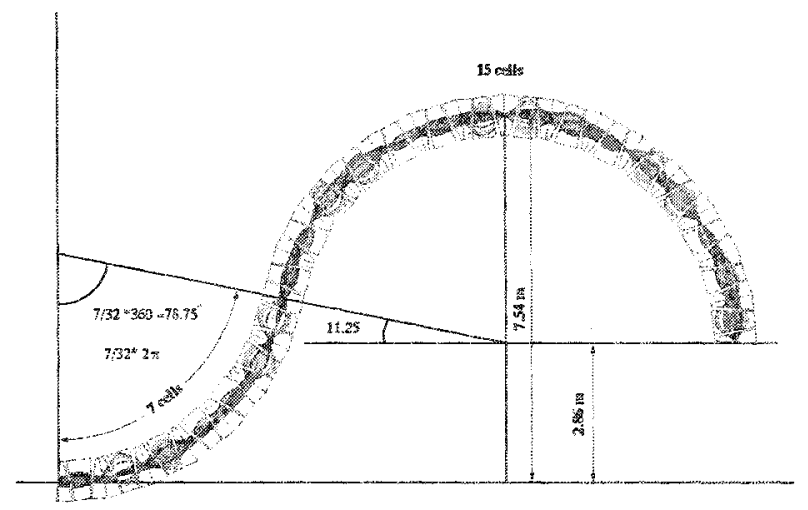

Fig. 4: A gantry made of 7 and 15 cells of the ring presented in previous figure. Orbit offsets are magnified 25 times. Tracking is performed with the PTC program.

\section{Particle Tracking in the Gantry}

Beam amplitudes within ellipses with maximum values for the horizontal axis of $x_{\max }=3 \mathrm{~mm}$ and $y_{\max }=3 \mathrm{~mm}$ are used as initial conditions for particle tracking through the gantry. Tracking results are shown in Fig. 5 and 6 for the $\mathrm{x}, \mathrm{x}$ ' and $\mathrm{x}$, $\mathrm{y}$ phase space.

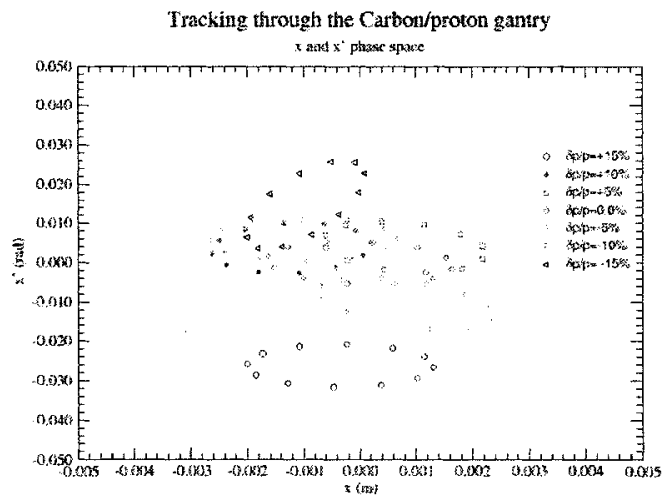

Figure 5: Tracking results in the $x$ and $x$ ' phase space.

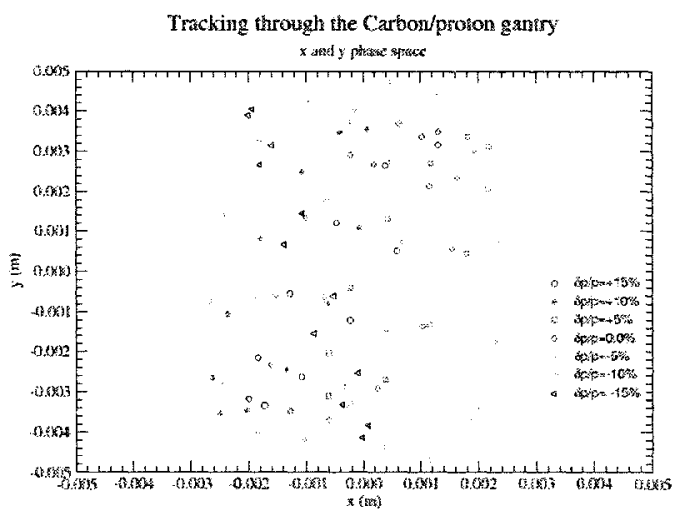

Figure 6: Tracking results in the $x-y$ plane. 


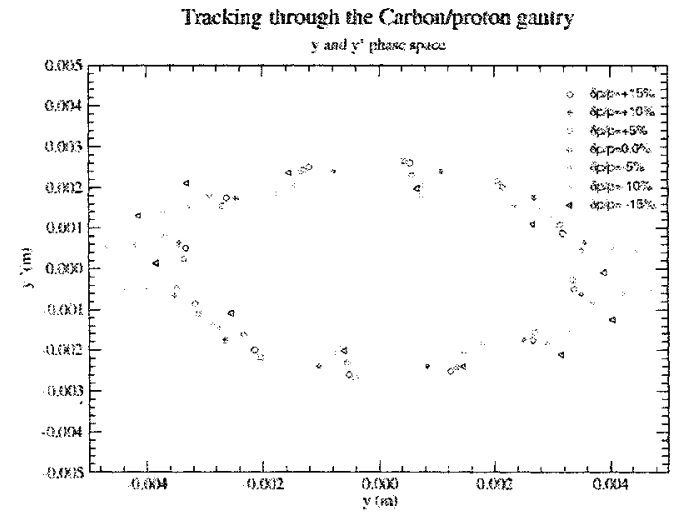

Fig. 7. Tracking results in the y,y' phase space.

\section{Beam Scanning}

The beam scanning should be of the order of $100 \mathrm{~Hz}$. The superconducting cables combined with the copper matrix could be even used in the beam scanning. Rough estimate is $50 \mathrm{~Hz}$.

\section{The size of the gantry's magnets}

There are about 24 to 30 non-scaling FFAG cells $(0.92$ meters long) used in the gantry design. The same number of major bends-combined function magnets is required. Magnet dimensions, field $B$, gradients $G$, and maximum aperture $A_{p}$, are summarized in Table 1.

Table 1: Magnet properties

\begin{tabular}{llllll}
\hline & $\mathrm{L}(\mathrm{m})$ & $\mathrm{B}(\mathrm{T})$ & $\mathrm{G}(\mathrm{T} / \mathrm{m})$ & $\mathrm{A}_{\mathrm{p}}(\mathrm{m})$ & $\mathrm{B}_{\max }(\mathrm{T})$ \\
\hline $\mathrm{BD}$ & 0.40 & $3.7-4.3$ & -68.5 & \pm .008 & 4.24 \\
$\mathrm{BF}$ & 0.40 & 1.00 & 71 & \pm .010 & 1.8 \\
\hline
\end{tabular}

The $40 \mathrm{~cm}$ long magnets of this size do not have excessive field requirements for superconducting magnets and could be built as the coil dominated magnetic field operating at lower temperatures $(2-4 \mathrm{~K})$. A configuration has a simple inner quadrupole surrounded by a thicker outer dipole coil and a very thin dipole coil (active shield) at much larger radius.

\section{Direct Wind Combined Function Gantry Magnet}

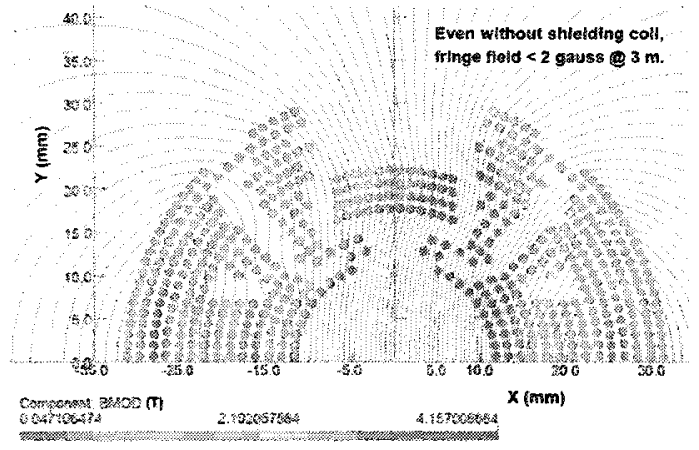

Fig. 8. Preliminary superconducting combined function magnet design.

\section{Direct Wind Combined Function Gantry Magnet}

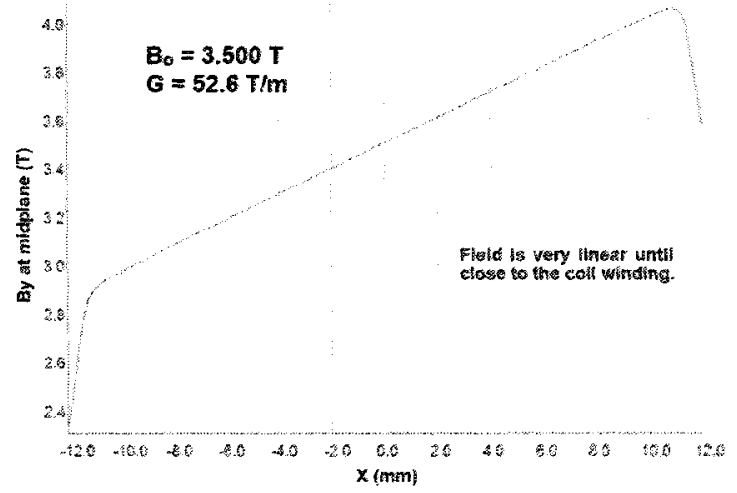

Fig. 9. Magnetic field along the transverse axis.

The cryostat has an $O D=170 \mathrm{~mm}$, and around the whole magnet is a thin warm iron shell to take care of the field not caught by shield coil. . The estimated weight of the magnets is in the range $45-75 \mathrm{~kg} / \mathrm{m}$ or average $\sim 50 \mathrm{~kg} / \mathrm{m}$. The $\sim 20$ meter long gantry beam line is $\sim 1400 \mathrm{~kg}$. This does not include the weight of the support system, but it is to be compared to the 135 tons of the "transport components" [4].

\section{SUMMARY}

An update on the non-scaling FFAG gantry, with two settings of the fixed magnetic field for the energy range of $135-400 \mathrm{MeV} / \mathrm{u}$ for the carbon-proton cancer treatment is presented. Small magnet sizes should reduce the cost and weight and ease the gantry operation. The transverse and final focus scanning system is assumed to be at end of the gantry transport above the patient. The carbon gantry size has been reduced from the previous [1] radius of $5.85 \mathrm{~m}$ to 4.6 , or the length of the transport system is reduced from $25.7 \mathrm{~m}$ to $20.2 \mathrm{~m}$ (number of cells is reduced from 28 to 22). The vertical distance from the end of the gantry to the patient bed is $2.86 \mathrm{~m}$.

\section{REFERENCES}

[1] D. Trbojevic, E. Keil, B. Parker, and A. M. Sessler, Phys. Rev. Spec. Topics-Accel. and Beams 10, 053503 (2007) pp. 1-6.

[2] U. Amaldi, Physica Medica - Vol. XVII, Supplement 1 (2001) 33-37.

[3] J. Lundkvist et. all., Acta Oncologica, 2005; 44, 850861.

[4] U. Weinrich, Invited talk at this conference.

[5] D. Trbojevic, E. Courant, and M. Blaskiewicz, Phys. Rev. ST Accelerator Beams 8, 050101 (2005).

[6] E. Forest et. al., CERN-SL-2002-044 AP. 